\title{
TIPOLOGÍAS Y MÉTODOS DE LA INTERVENCIÓN POÉTICA. UN BREVE ACERCAMIENTO DESDE LA ESTÉTICA
}

Jordi CLARAMONTE ARRUFAT

UNED

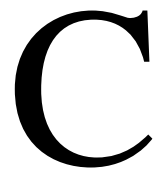

on los años y las modas culturales se ha ido imponiendo una especie de escisión entre las investigaciones derivadas del campo de la Estética -concebida como teoría de la sensibilidad y de las artes- y los estudios literarios. Con todo, no creemos que la distancia entre ambos campos deba ser mucho mayor que la que recomienda la autonomía relativa de cualquier desarrollo disciplinario. Por eso, y porque por nuestra parte damos en reclamar una tradición que tiene a Schiller, Nietzsche, Benjamin o Lukács entre sus mejores representantes, creemos que cabe esperar fértiles interacciones entre estos dos ámbitos de investigación y confiamos en que este artículo pueda, al menos en parte, demostrarlo.

Más en concreto, en este breve trabajo quisiera darme la oportunidad de proponer una tipología de las intervenciones artísticas dotadas de un cariz explícitamente político y orientadas a su despliegue en espacios públicos, en la confianza de que esta misma tipología pueda resultar de interés para estudiar mejor el funcionamiento de las intervenciones propiamente poéticas en diversas esferas públicas. Se tratará, como es obvio, de una tipología que no se pretende exhaustiva ni totalizante. Antes al contrario, desde el principio hay que declarar que se trata de una clasificación por completo interesada: del todo conveniente a mis intereses teóricos y prácticos, que no son otros que los de elucidar las posibilidades, las potencias y las impotencias de este orden de prácticas artísticas.

A tal fin puede resultar fértil definir tres tipos diferentes de prácticas que muestran diferentes flaquezas en sus métodos y despliegues. Trataremos brevemente de los siguientes tres tipos: las poéticas de la denuncia, las poéticas colaborativas y las poéticas relacionales. Vayamos, pues, por partes. 
Tipologías y métodos de la intervención poética...

\section{Poéticas de la denuncia}

Podríamos denominar poéticas de la denuncia a aquellas prácticas que, entendiéndose comprometidas social y políticamente, siguen mostrando buena parte de las características del arte de autor, volcadas en lo fundamental a la producción de obra mediada por un artista convenientemente identificado y acreditado que cuida la factura formal y la presentación de su trabajo. La poética de denuncia se caracteriza por tomar determinados aspectos socialmente problemáticos e introducirlos en su despliegue de modo más o menos directo. Se suele entender de qué "trata" la obra en cuestión, y el espectador por lo general cree haber entendido la obra en cuanto ha captado el mensaje que esta conlleva. Por lo demás, este tipo de práctica casi siempre se produce y se distribuye a través de los cauces habituales del mundo de la cultura y el arte: grandes medios de comunicación, museos, ferias de editores, etc. sin cuestionar la idoneidad de dichos cauces, ni siquiera su abierta complicidad con los males denunciados. Algunos artistas de este género, como Hans Haacke, han hecho trinchera de tales contradicciones. Así su proyecto sobre la Obra Social de La Caixa (Grasskamp et al., $1995)$ presentado en la Fundació Tàpies. Por lo general, no obstante, lo más normal es que las muestras de arte político, en las que el MACBA ha sido especialmente prolijo, se concentren en exponer muestras de arte político lejano, ya sea en el tiempo ya sea en el espacio.

Dada la repercusión mediática y el prestigio que tienen los eventos producidos en semejantes circuitos, la "causa" elegida por las poéticas de denuncia suele, cuando menos, alcanzar cierta notoriedad o salir del olvido en el que acaso estaba. Así, las denuncias y fakes de los YesMen sobre la catástrofe de Bophal (The YesMen, 2004), por ejemplo.

El caso es que estos autores - pienso en Santiago Sierra, por ejemplo- rara vez articulan su productividad con una comunidad o una red social de afectados por la denuncia realizada en su obra; con lo cual, obviamente, la efectividad mediática de la intervención no suele ir acompañada de una transformación de la capacidad de agencia social, ni a corto ni a largo plazo de dicha red. Diríase que buena parte de este orden de intervención poética tiene la virtualidad de exponer determinado conflicto pero lo hace de tal modo que éste comparece en un vacío agencial, desvinculado por así decir de las gentes que le dieron o dan cuerpo y continuidad. Es obvio que esto puede producir una recepción deformada, acaso estetizada, de la conflictividad social que se pretendía tratar $^{1}$.

\footnotetext{
${ }^{1}$ Para un estudio en profundidad de las implicaciones entre las prácticas politizadas del arte y la noción de esfera pública puede verse Blanco et al. (2001), especialmente la tercera sección.
} 
Por lo demás, los autores que intervienen en estas coordenadas suelen tener una comprensión de lo político delimitada por las "grandes cuestiones": la inmigración, la memoria histórica, el colonialismo..., dejando de lado cuestiones de índole más cotidiana y más humildes, que sin duda recabarían menor atención de los medios o harían parecer menos radicales las propuestas de tan esforzados artistas.

En conclusión: parece que a la eficacia mediática de estas prácticas no suele acompañarla ni la articulación social y política real ni una especial sutileza para captar los niveles en que opera lo político en las sociedades del capitalismo tardío.

\section{Poéticas colaborativas}

Con esto podemos pasar a considerar ahora un segundo tipo de intervención poética que denominaremos "poéticas colaborativas". Situadas quizás en las antípodas institucionales de las anteriores, se localizan en un abanico de prácticas que pretenden articularse estrechamente con redes y movimientos sociales, procurando dotarlos de herramientas expresivas o comunicativas más potentes y afinadas.

Estas prácticas suelen priorizar el proceso mismo de colaboración e incluso de producción de las redes sociales en que se desarrollan, pudiendo descuidar considerablemente lo que en términos tradicionales sería la obra en tanto "resultado" estabilizado de ese proceso, pudiéndose llegar en ocasiones a cierta displicencia en este plano, lo cual llega a restar a veces peso específico al conjunto de la intervención. Agencias o Bordergames (Claramonte, 2011) es un buen ejemplo de ese problema por el que la priorización del proceso puede conducir a que las herramientas generadas pierdan precisamente la potencia y la especifidad que les daba su razón de ser. La solución a esta contradicción no es fácil en absoluto y sólo mediante un trabajo continuado en el tiempo es posible acaso generar en las redes con las que se trabaja un aprecio por las herramientas que se aportan. El trabajo de El Lobby Feroz en el "parque de la muy disputada cornisa" en Madrid (Claramonte, 2011), en el que se logró socializar todo un lenguaje específico y todo un nivel de intervención podría ser un ejemplo interesante a ese respecto.

Obviamente, estas prácticas pueden producir redes sociales potentes o reforzar las ya existentes pero rara vez alcanzan la notoriedad de las prácticas reseñadas más arriba, sobre todo si han de suceder en ciclos largos de tiempo. Por lo demás, y dadas las limitaciones que se autoimponen en función de su articulación política, no es extraño que su ámbito de trabajo se vea limitado a una serie de cuestiones políticamente correctas para el círculo de los movimientos en los que se integran, careciendo de capacidad para alterar o ampliar de modo significativo la agenda política establecida en 
Tipologías y métodos de la intervención poética...

cada caso. Esta es sin duda, una limitación que el "arte colaborativo" comparte con el "arte de denuncia", en la medida en que a menudo se trata de prácticas artísticas que mantienen una visión muy limitada sobre cuáles sean los espacios susceptibles de intervención política, limitándose en consecuencia a actuar en los dominios reconocidos como políticamente significativos. La consecuencia es el descuido de los niveles que cabría perfilar en términos de micro-política o biopolítica. Y precisamente ésos son los niveles en los que se mueve el tercer tipo de arte político al que aludiremos.

\section{Poéticas relacionales}

En efecto, consideraremos ahora las intervenciones poéticas cercanas por su concepto a lo que se ha dado en denominar "arte relacional": toda una constelación de prácticas que en gran medida derivan de la ampliación tanto del ámbito de lo artístico operado por las últimas neovanguardias, de Fluxus a la IS, como del ámbito de lo político definido por el advenimiento del "capitalismo cultural". Jameson trama este concepto desde su análisis de la postmodernidad, que, según señala, «no se puede separar ni pensar sin la hipótesis de una mutación fundamental de la esfera de la cultura en el mundo del capitalismo tardío, mutación que incluye una modificación fundamental de su función social» (Jameson, 1991: 66). En la postmodernidad, que viene a coincidir con lo que Mandel denominaría fase de capital multinacional, «el proceso de modernización ha concluido, la naturaleza se ha ido para siempre... la cultura se ha convertido en una auténtica segunda naturaleza» (Jameson, 1991: 10).

Esta fase multinacional del capitalismo se da así en el momento en que su equilibrio y proliferación ha pasado a depender en grado creciente de su reproducción en los ámbitos de la vida cotidiana. Ahí nos encontramos con que no se trata ya de vender mercancías, sino modos de vida, culturas:

De este modo en la postmodernidad la cultura se ha vuelto un producto por derecho propio [...]. La postmodernidad es el consumo de la pura mercantilización como proceso [...]. Así pues el "estilo de vida" del superestado guarda la misma relación con el "fetichismo" de las mercancías de Marx que los monoteísmos más avanzados con los animismos primitivos.

Tenemos que ser capaces de vérnoslas entonces con un concepto de lo cultural que no sea una especie de accesorio, complemento más o menos espurio de las ideas explícitamente políticas o de las posiciones estructurales de sujeto:

[...] la disolución de una esfera autónoma de la cultura debe más bien imaginarse en términos de una explosión: una prodigiosa expansión de la cultura por el ámbito social, hasta el punto que se puede decir que todo lo que contiene nuestra vida social -desde el valor económico y el poder estatal hasta las prácticas y la propia estructura mental- se ha 
vuelto "cultural" en un sentido original y que todavía no se ha teorizado (Jameson, 1991: 72).

Diríase que las intervenciones poéticas de corte relacional se han especializado en señalar y articular niveles de intervención y agencialidad política que tienen que ver con esa importantísima redefinición de lo cultural, redefinición que convierte lo cotidiano, lo convivencial, los lenguajes y las pequeñas sociedades en las que nos movemos y hacemos, en nichos políticamente relevantes, urgiéndonos a trabajar en el cuestionamiento y la rearticulación de esos mismos ámbitos.

Curiosa y yo diría que lamentablemente, la mayor parte de estas prácticas se han circunscrito muy pronto, y de modo casi fundacional si atendemos a teóricos como Nicolas Bourriaud (1998), a su despliegue en el seno de los circuitos más tradicionales del mundo del arte, redefinidos ahora -no sin una cierta complacencia- como nuevos laboratorios de experimentación social.

Con ello el arte relacional no ha conseguido ni la efectividad mediática del "arte de denuncia" ni la articulación social efectiva del "arte colaborativo".

\section{Conjunción de la autonomía y la funcionalidad social del arte}

Nos encontramos pues con que ninguna de las tres variantes de intervención poético-política vistas puede sentirse satisfecha de haber desplegado una agencialidad transformadora digna de consideración. Y lo que es más importante, vamos teniendo cierta idea además de las carencias que han ayudado a que así fuera. Si bien cada una de ellas muestra alguna vertiente en la que se la puede juzgar de manera favorable, se diría que sus puntos flacos las desarbolan en tanto prácticas efectivas en términos políticos y artísticos. Siendo así, en tanto no tengamos la capacidad de pensar una práctica que reúna vigor formal, articulación social y apertura para concebir los niveles en los que actúa lo político, no se podrá hablar propiamente de intervenciones poéticas en el espacio público concebidas como forma de arte político. No será posible usar los dos términos - "arte" y "político"- con plena legitimidad. Tendremos que seguir considerando las vanguardias como repercusiones imaginarias de explosiones que nunca han estallado.

Con este fin, deseo plantear los lineamientos de al menos dos elementos que me parecen de la mayor relevancia a la hora de concebir prácticas de intervención poética en la esfera pública de un modo quizá algo más ambicioso al visto hasta aquí.

El primero es la diferenciación y la especifidad de lo artístico, que creo que hay que vindicar con suficiente claridad. Se trata de uno de los fundamentos de la 
Ilustración y de la Modernidad, surgido de los procesos de diferenciación de las facultades humanas, por los cuales se especifica un "modo de reacción" específicamente estético, del mismo modo que se hizo lo propio con el modo de reacción diferenciado que está en las bases del pensamiento científico. Ese modo de reacción estético podría caracterizarse, al decir de un pensador tan poco sospechoso de esteticismo como Lukács, por su capacidad de generar un medio homogéneo, una unidad tonal emocional, que se separa de lo que este teórico describió como el gran río de la vida cotidiana a fin de constituirse en su especifidad y alcanzar así -lo dijo Lukács (1982, vol. II, 350 y ss.) - su forma más pura antes de acabar volviendo al río aquel de la vida cotidiana.

Sólo teniendo presente esta capacidad, tradicionalmente asignada al arte y ahora disuelta en el campo de la percepción estética general, podremos concebir un ámbito de alteridad relacional, una lógica perceptiva y distributiva diferente de la que rige la economía de mercado de la vida cotidiana. Toda propuesta que quiera cuestionar las distribuciones relacionales y situacionales que constituyen lo establecido en toda su violencia debe ser capaz de apartarse de la lógica de lo dado, de los bloques de lo instituido. Debe sugerir diferentes modalidades de relación con los objetos y aportar sensibilidades específicamente diferenciadas.

Ahora bien, si esta especifidad de lo estético constituye por sí sola un ingrediente imprescindible del funcionamiento político del arte, en la medida en que hace visible la posibilidad misma de la alteridad relacional, es evidente que no se basta para definirlo. Será por tanto preciso que consideremos un segundo elemento al que denominaremos capacidad de despliegue en lo cotidiano. Ya presente en Schiller o en Nietzsche, hablamos en definitiva de la concepción que relaciona lo estético con las posibilidades ya no de la percepción estética -situada por ello mismo en un contexto netamente diferenciado- sino con las de distribución y organización de la vida cotidiana misma. De Nietzsche a Foucault se ha hablado de una suerte de bioestética que en sus versiones más interesantes no se limita a barajar y reorganizar las posibilidades perceptivas del sujeto hegemónico, sino que cuestiona este sujeto, transformando el orden ontológico que supuestamente lo funda en una posibilidad modal más. Lo que las prácticas artísticas y la percepción estética constituyen en su modo de reacción específico debe codificarse de manera tal que pueda ser transformado en una lógica relacional, en un modo de hacer.

Estos dos elementos que se han visto, autonomía y funcionalidad social, fueron tratados por la mayor parte de las teorías del arte como tendencias antitéticas, cuando no mutuamente excluyentes. Por nuestra parte, y desde la apuesta partisana por la potencia de las intervenciones poéticas en los espacios públicos, nos hemos visto en la necesidad de pensar recursos teóricos que permitan concebir juntamente ambos vectores. Cuando 
reunimos en un único dispositivo teórico la especifidad del modo de reacción estético y su funcionamiento en tanto diagrama relacional abierto nos encontramos con una nueva síntesis que hemos denominado modo de relación.

Los modos de relación constituyen gramáticas específicas de organización de la percepción, la representación y el comportamiento en tanto éstos -insistimos- no son reducibles a concepto. Parafraseando a Foucault podría sostenerse que al decir modo de relación aludimos a una actitud con y frente a la actualidad, a

una manera de pensar y de sentir, de actuar y de conducirse que marca una relación de pertenencia y, simultáneamente, se presenta a sí misma como una tarea. Un poco, sin duda, como aquello que los antiguos griegos denominaban un ethos... (Foucault, 1984; la traducción es nuestra).

De esta forma, los modos de relación son siempre determinadas intensidades específicas, determinadas modulaciones de las relaciones que se establecen entre los artistas, los espectadores y el medio en que todos ellos se hallan, y esto es así de modo tal que incluso cuando "la obra" se ha acabado ésta puede rememorarse en función de lo que ya podemos llamar su entidad modal: su específica consistencia a la hora de definir $\mathrm{y}$ articular un conjunto de relaciones ${ }^{2}$ que definen y establecen las subjetividades $\mathrm{y}$ objetividades puestas en juego a cada momento.

\section{Lo repertorial, lo disposicional, el paisaje}

Otra forma de abordar esta encrucijada conceptual en la que estoy situando la específica forma de la efectividad política y social de las prácticas artísticas, y con ellas también las intervenciones poéticas, sería considerar cómo los modos de relación surgen del cruce de un nivel repertorial con uno disposicional sobre un paisaje concreto.

Entendemos lo repertorial como un conjunto relativamente estable de formas "primas" que dan cuenta del abanico de posibilidades que, en un momento dado, nos define como cultura o incluso (cuando nos ponemos marxianos) como especie. El polo de lo repertorial pretende dar cuenta de esa "relativa estabilidad", de esa constelación de formas que tienden a permanecer y que debemos evitar llamar identidad para no

\footnotetext{
${ }^{2}$ Podemos encontrar claros precedentes de estructuración modal en buena parte, si no en la mayoría, de las estéticas premodernas y no occidentales, cuyas distribuciones del material artístico y las posibilidades de experiencia estética se agrupan invariablemente alrededor de conceptos de clara índole modal -como el hindú raga- y que aluden no solo a determinadas escalas musicales, por seguir con el ejemplo, sino también e indisociablemente al conjunto de circunstancias ambientales y humanas que rodean y hacen posible y pensable dicho raga. En todas estas estéticas, por tanto, las unidades básicas que nos permiten pensar tanto la producción como la recepción estética no se restringen en torno al polo del "artista" productor, ni al del movimiento artístico, ni al del estilo siquiera, en que dicha experiencia se genera.
} 
agarrotarla, pero a la que sí podemos reconocerle, necesariamente, una cierta remanencia.

El nivel disposicional, por su parte, puede entenderse como elucidación generativa de lo repertorial, como conjunto de competencias, de inteligencias específicas que actualizan y despliegan de modos siempre diferentes las posibilidades contenidas en lo repertorial.

El polo de lo disposicional por tanto, comparece como necesaria intervención discreta sobre lo repertorial, como articulación que lo actualice, que lo ponga a definirse. Como sucedería con una música o una épica que desde una base rítmica o temática compartida (nivel repertorial) se tiene que ejecutar, improvisar performativamente (nivel disposicional) cada vez que se toca o se oye.

Finalmente, pensamos el paisaje como una matriz de conflictividades posibles, donde diversos modos de relación pertenecientes o no a una misma repertorialidad han de convivir. En muchos casos -y con la globalización, cada vez más- lo que el paisaje alberga son choques y encuentros de fragmentos, añicos casi, de diferentes repertorialidades y diferentes organizaciones de lo disposicional. A menudo, reitero que cada vez más, los repertorios aparecen fragmentados y las competencias que dan pie a nuestra disposicionalidad surgen extrañadas, convertidas en triste excepción o en patrimonio corporativo de expertos.

La introducción de estos niveles en la discusión nos permite pensar ahora diferentes intensidades y direcciones en las que pueden desplegarse las intervenciones poéticas que constituyen nuestro objeto de reflexión. Así una intervención poética tendrá un carácter repertorial cuando tienda a (re)constituir un conjunto coherente de formas de la sensibilidad que dé cuenta del modo más completo y más claro posible de aquello que nos afecta y nos define, ya sea como cultura, como tribu o como cualquier otro orden de comunidad. Una intervención poética tendrá una orientación disposicional cuando se dirija a poner de manifiesto, a rehabilitar o a instituir las competencias e inteligencias específicas que nos constituyen como agentes linguiísticos, como sujetos de sensibilidad y dispositivos de acción. Finalmente, toda intervención poética en y sobre el espacio público deberá dar cuenta de una u otra manera del paisaje en que va a desplegarse, mostrando cuáes son los límites de tolerancia del mismo, qué modos de relación han sido excluidos o cuáles ni siquiera han tenido nunca cabida.

\section{La intervención poética en el espacio público}

Un tanto pretenciosamente, he llamado estética modal al conjunto de tesis que he estado desarrollando en los últimos años para pensar estas cuestiones. La estética modal 
concibe que toda obra de arte, toda intervención poética, consta de un centro, de una forma formante que convierte su organización formal en propuesta distributiva, modo de vida, de deseo; modo de relación, en suma, de las diferentes partes postuladas por la obra y de este conjunto con el resto del mundo

Al igual que la mayoría de las estéticas previas a Hegel y el Romanticismo, la estética modal no asume como objeto disciplinar natural el restringido campo de las prácticas reconocidamente artísticas. Va más allá para abarcar lo que hay de común entre éstas y el amplio campo de experiencias e ideas que, en su no ajustarse a concepto, cabe denominar, con plena legitimidad, estéticas.

En consecuencia, llamaremos intervención poética en el espacio público al proceso por el que un grupo de gente construye las condiciones concretas para un ámbito de libertad concreta liberando, al hacerlo, un modo de relación.

\section{Referencias bibliográficas}

BLANCO, P. et al., eds. (2001): Modos de hacer: arte crítico, esfera pública y acción directa. Salamanca, Universidad de Salamanca.

BOURRIAUD, N. (1998): Estética relacional. Barcelona, Paidós, 2006.

CLARAMONTE, J. (2011): Arte de contexto. Donosti, Nerea.

FOUCAULT, M. (1984): “Qu'est-ce que les Lumières?”, en http://www.foucault.info/ documents/whatIsEnlightenment/foucault.questcequeLesLumieres.fr.html (última consulta, 4-12-2011).

GRASSKAMP, W. - HAACKE, H. - BUCHLOH B. (1995): Obra social: Hans Haacke. Barcelona, Fundació Antoni Tàpies.

JAMESON, F. (1991): Teoría de la postmodernidad. Madrid, Trotta, 2001.

LUKÁCS, G. (1963): Estética. Barcelona, Grijalbo, 1982.

THE YESMEN (2004): The True Story of the End of the WTO. Nueva York, The Disinformation Company. 